\title{
Selective attention and asymmetry in the Müller-Lyer illusion
}

\author{
JOHN PREDEBON \\ University of Sydney, Sydney, New South Wales, Australia
}

\begin{abstract}
Two experiments reexamined the effect of selective spatial attention on the magnitudes of the wingsin and wings-out forms of the Müller-Lyer (M-L) illusion and a version of the illusion in which the two forms are superimposed to produce a figure (XX) flanked at both ends by an $X$. For the XX figure, ignoring the outer wings produced significant underestimation of shaft length, whereas ignoring the inner wings had no significant effect. For the M-L figures, ignoring the wings was more effective in attenuating the magnitude of the wings-out than of the wings-in illusion. The results are discussed with reference to space-based approaches to visual attention and to claims that attentional modulation of illusion magnitudes implicates high-level or cognitive factors in the formation of the M-L illusion.
\end{abstract}

Partly because of its exemplar status for demonstrating the mismatch between "reality" and perception that can occur in everyday visual processing, and partly because of its potential for understanding the processes of normal perception, the Müller-Lyer (M-L) illusion continues to attract empirical and theoretical interest. The illusion consists of two forms, a wings-in or underestimated form and a wings-out or overestimated form (Figures 1A and $1 \mathrm{~B}$, respectively), although it is also studied in its combined form (Figure 1C).

It is now generally accepted (e.g., Coren \& Porac, 1983; Restle \& Decker, 1977) that the M-L illusion is a resultant of several factors interacting at different levels of visual processing, ranging from low-level mechanisms, such as optical blur and retinal inhibition, to highlevel mechanisms, such as cognitive contrast and visual attention. This report considers the role of visual attention in modulating the magnitude of the M-L illusion.

Two approaches have been used to examine the effect of selective spatial attention on M-L illusion magnitudes. One approach is to vary the distribution of spatial attention across the components of the M-L figure, as in Gardner and Long's (1961) study, which found that attending to the shaft and ignoring the wings attenuated the magnitude of the combined M-L illusion. As was noted by Goryo, Robinson, and Wilson (1984), however, this approach cannot distinguish between the "external" and "internal" determinants of selective attention - that is, whether the reduction is effected by changes in visual input consequent on selectively foveating on different parts of the figure (an external effect) or by modulations of the processes performed on the input (an internal effect).

The other approach (Rock, 1975), which uses the superimposed or XX version (Figure 1D) of the two M-L

Correspondence concerning this article should be addressed to J. Predebon, School of Psychology, University of Sydney, Sydney, NSW, 2006, Australia (e-mail: johnp@psych.usyd.edu.au). forms, assesses the effect of ignoring either the inner $(<>)$ or the outer $(><)$ wings of the XX figure on judgments of the shaft's length. Since both M-L forms are present in the same spatial region, this approach is believed to eliminate possible differences in foveation to the two M-L components, thereby allowing an assessment of the impact of selective attention on the internal processes responsible for the formation of the M-L illusion. The results of the three relevant studies (Coren \& Porac, 1983; Goryo et al., 1984; Tsal, 1984) show that selectively attending to either the inner or the outer wings produces distortions of shaft length in the direction of the attended M-L component, an outcome seemingly implicating high-level cognitive mechanisms in the formation of the M-L illusion.

A consistent outcome of the XX studies is the asymmetry of the attentional effects: Whereas ignoring the outer wings yields substantial underestimation of shaft length, ignoring the inner wings produces only a small overestimation (Coren \& Porac, 1983; Goryo et al., 1984) or no effect at all (Tsal, 1984: Experiment 1), suggesting that it is easier to ignore the outer than the inner wings. Only Tsal (1984) directly addressed this intriguing finding. He argued that the difficulty in ignoring the inner wings while attending to the outer wings might be due to the inner and outer wings forming coherent figures (i.e., $X \mathrm{~s}$ ), which may have rendered the individual wing components of the $X$ s difficult to segment perceptually. Although segmentation difficulty may have been a factor in Tsal's Experiment 1, which used black-and-white figures, it is unlikely to explain the asymmetry found by either Coren and Porac (1983) or Goryo et al. (1984), since both of these studies used color differences to perceptually distinguish the inner from the outer wings. In any case, if segmentation difficulty was the primary factor in Tsal's work, then it should have been equally difficult to ignore the outer as to ignore the inner wings, in which case the XX illusion asymmetry is puzzling. 
A

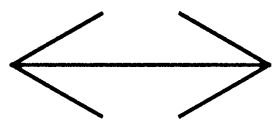

C

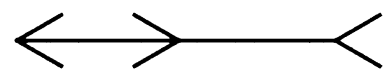

B

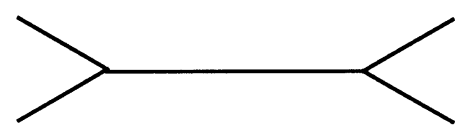

D

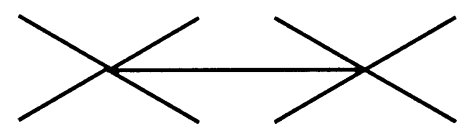

Figure 1. The wings-in or underestimated (A) and wings-out or overestimated (B) forms of the Müller-Lyer illusions; the combined (C) and the superimposed or XX (D) versions of the Müller-Lyer illusion.

Contemporary research distinguishes between objectbased approaches, in which attention selects objects or groups of objects, and spatial-based approaches, in which attention selects a region of space (see Scholl, 2001, for a review). A version of the segmentation hypothesis, an object-centered approach to attention, could account for the asymmetry of the attentional effects in the XX studies if the inner wings are more likely than the outer wings to form a natural perceptual entity or grouping with the shaft, perhaps because of the closer spatial proximity of the inner wings to the shaft. However, the asymmetry is also consistent with certain spatial-based theories of visual attention, such as the zoom-lens theory (Eriksen \& St. James, 1986) and Pressey's (e.g., Pressey, 1971; Pressey \& Martin, 1990) attentive-field theory of visual size illusions. According to Pressey's theory, events falling within the attentive field are given privileged processing, with the focal extent averaged or integrated to the other explicit and implicit contextual extents within the attentive field. The theory assumes that the deployment of the field in a visual display and the field's size are determined to a large degree by the nature of the observer's task. In the case of the XX figure, for example, the deployment of spatial attention is determined mainly by the end points of the focal extent (i.e., the shaft), not by the contextual extent (i.e., the overall length of the XX figure). As the shaft length defines the minimum diameter of the attentive field, it should be easier to ignore the outer than the inner wings, thus yielding the illusion asymmetry found in the XX studies.

One aim of the present investigation was to confirm the asymmetry of the attentional effect on XX illusion magnitudes using color to differentiate the inner from the outer wing components, as in Coren and Porac's (1983) and Goryo et al.'s (1984) studies, but with the addition of a plain-line control. Unfortunately, the XX studies did not include a control condition against which to evaluate the magnitudes of the under- and overestimations of the XX figure, referencing the estimations instead to the actual length of the figure's shaft. Comparing the estimations to the actual length of the shaft, however, is valid only if observers accurately judge the lengths of nonillusory extents (i.e., control lines). However, if observers underestimate the length of a control line of the same length as the shaft of the XX figure, then the asymmetry between the over- and underestimation magnitudes will disappear or even reverse, depending on the extent of the underestimation. The XX illusion asymmetry therefore could be an artifact of the measurement procedure and might not be evident if the length judgments are referenced to appropriate baseline measures. For this reason, the present investigation quantified M-L effects by subtracting subjects' length reproductions of a plain line from their reproductions of the M-L figures.

The other aim was to extend the investigation to the wings-in and wings-out M-L illusion forms. According to the space-based attentional accounts, concentrating attention on the shaft should produce a greater proportional reduction in the magnitude of the wings-out than of the wings-in M-L illusion, simply because it is easier to ignore the wings of the wings-out than of the wingsin M-L figure. In contrast, since the wings of both M-L forms are easily perceptually segregated from their shafts, the segmentation-difficulty hypothesis predicts similar proportional reductions in the magnitudes of the two illusion forms. Surprisingly, no study has examined the effect of ignoring the wing components of the wings-in and wings-out M-L illusion forms separately. The present investigation therefore provides a novel and straightforward test of the predictive utilities of the space-based and segmentation-difficulty accounts of the XX illusion asymmetry.

\section{EXPERIMENT 1}

Preliminary work using M-L figures with differentcolored wings and shafts indicated that prior experience with the task of ignoring the wings made it difficult for subjects to subsequently adopt a neutral set or attitude in judging the length of the shaft of same-colored M-L figures. As it was necessary in Experiment 1 to compare the relative magnitudes of the $\mathrm{XX}$ and $\mathrm{M}-\mathrm{L}$ illusions under both neutral and selective-attention conditions, this problem was minimized by using black-and-white stimuli for the neutral conditions and by presenting the neutral and attentional trials in separate blocks.

\section{Method}

Subjects. The subjects were 16 volunteer undergraduate students. All had normal or corrected-to-normal vision. 
Stimuli and Apparatus. There were two sets of stimuli, a test or colored set and a baseline or black set. The test set contained a horizontal plain control line and eight horizontal M-L figures formed by factorially combining two wing angles $\left(60^{\circ}\right.$ or $90^{\circ}$, i.e., the internal angle between the end obliques), and four M-L forms (the wings-in and wings-out M-L figures and two XX figures). The lengths of the horizontal and oblique lines were 50 and $20 \mathrm{~mm}$, respectively. For the M-L figures, the wings and the shaft were in different colors. For one XX figure, the outer wings and shaft were the same color and the inner wings a different color. For the other XX figure, the inner wings and shaft were the same color, and the outer wings a different one. There were two versions of the test figures. For one version, the horizontal lines were blue, as were the outer wings of one of the XX figures and the inner wings of the other; all the other wings were red. For the other version, the colors were interchanged: The horizontal lines were red, as were the outer wings of one of the XX figures and the inner wings of the other, and all the other wings were blue. The baseline set of stimuli was identical to the test stimuli, except that all lines were black and there was only one XX figure.

The figures were presented in the upper left of a gray-white $25 \times$ $35 \mathrm{~cm}$ computer screen together with a horizontal black comparison line in the lower right quadrant. All lines were two pixels wide (approximately $0.6 \mathrm{~mm}$ ). Subjects could vary the length of the comparison line by moving the computer mouse to the right or left to shorten or lengthen the line's length, respectively. The computer recorded the reproduction when the subject signaled completion by pressing any key on the computer keyboard. During 10-sec intertrial intervals, the monitor was blank. Participants viewed the stimuli with free inspection from a distance of $57 \mathrm{~cm}$ while resting their chins on a chin and head restraint device. From this distance, the shaft and oblique lines subtended visual angles of $5^{\circ}$ and $2^{\circ}$, respectively.

Procedure. The test and baseline stimuli were presented in separate blocks, with the block order counterbalanced across subjects. The interblock interval was approximately $30 \mathrm{~min}$. Subjects were given practice with the reproduction task using 40- and 60-mm-long lines.

Test session. Half the subjects were given the blue, and the other half the red horizontal-line stimulus sets. Colored drawings of the figures were used to demonstrate the attentional task for each type of stimulus figure. The initial instructions required subjects to pay close attention to one set of colored lines (e.g., red wings and red shaft) and to completely ignore the other colored lines (e.g., blue wings). Subjects were then positioned in the viewing device and instructed, once again, to attend to the red (or blue) lines and to completely ignore the blue (or red) ones. Each subject received two randomized presentations of the seven stimuli. Subjects made two counterbalanced judgments of a test figure, one with an ascending and the other with a descending starting position, before proceeding to the next figure.

Baseline session. This was similar to the test session except for differing instructions and the use of black-and-white stimuli. The instructions directed subjects to look at the figure without ignoring or selectively attending to any part of the figure and to base their judgments on their visual impressions of the shaft's length.

Notation. The following notation is used to describe the XX conditions: XX-neutral (black figure, no selective attention); XX-out (colored figure, attend to the outer and ignore the inner wings); $\mathrm{XX}$-in (colored figure, attend to the inner and ignore the outer wings).

\section{Results}

The judgments of the 50-mm-long control line of the test and the baseline stimulus sets, which were used to estimate each subject's point of subjective equality (PSE), were $-.45(S E=.57)$ and $-.34(S E=.69) \mathrm{mm}$, respectively. Neither mean is significantly different from zero $[t(15)=-0.78$ and -0.49 , respectively; $p \mathrm{~s}>.05]$. Each subject's judgments of the M-L figures were converted to deviations from PSE by subtracting the PSE from the reproductions of each of the test figures. Positive and negative scores indicate over- and underestimation of shaft length, respectively. Since the initial analysis indicated that neither the order of block presentation nor the type of colored stimulus set had a significant effect, the data were combined across these two factors. The results of all conditions are shown in Figure 2.

XX results. The $60^{\circ}$ and $90^{\circ} \mathrm{XX}$-neutral conditions (Figure 2A) yielded accurate length matches: Neither mean was significantly different from zero $[t(15)=$ -0.63 and 0.37 , respectively; $p \mathrm{~s}>.05$ ]. The results of the attentional conditions (Figure 2B) indicated an effect of selective attention for the XX-in but not for the XXout figures. The XX-in conditions produced significant underestimations of shaft length for both the $60^{\circ}$ and $90^{\circ}$ figures $[t(15)=3.06$ and 2.15, respectively; $p \mathrm{~s}<.05]$, indicating that subjects were able to ignore the outer and attend to the inner wing components. In contrast, for neither the $60^{\circ}$ nor the $90^{\circ}$ figures of the XX-out condition was the error significantly different from zero $[t(15)=$ 0.48 and 0.44 , respectively; $p \mathrm{~s}>.05$ ], indicating that subjects were unable to ignore the inner and attend to the outer wing components.

M-L results. The results of the baseline or neutralattention conditions (Figure 2A), confirmed the usual M-L effects: Both the $60^{\circ}$ and $90^{\circ}$ wings-in figures were underestimated, and both the corresponding wings-out figures were overestimated $[t(15)=11.94,7.67,12.42$, and 11.23 , respectively; $p s<.05]$. The magnitude of the M-L illusion tends to be greater for smaller wing angles (e.g., Erlebacher \& Sekuler, 1969), and this effect was also evident in the data presented in Figure 2A. For the wings-out illusion, the difference between the $60^{\circ}$ and $90^{\circ}$ conditions was significant $[t(15)=2.84, p<.05]$. The corresponding difference for the wings-in illusion, however, was marginally nonsignificant $[t(15)=2.04$, $p=.059]$. The absolute magnitude of the wings-out M-L illusion typically is greater than that of the wings-in illusion (e.g., Christie, 1975), and a similar asymmetry of illusion magnitudes can be seen in Figure 2A: Averaged across the angle factor, the magnitude of the wings-out illusion was 2.2 times greater than the magnitude of the wings-in illusion.

The results of the selective-attention conditions (Figure $2 \mathrm{~B}$ ) indicated that subjects were unable to ignore completely the wing components of the wings-in and wings-out figures. The underestimation of both the $60^{\circ}$ and $90^{\circ}$ wings-in figures was significant $[t(15)=7.97$ and 8.06, respectively; $p \mathrm{~s}<.05$ ], as was the overestimation of the wings-out figures $[t(15)=12.51$ and 10.39 , respectively; $p \mathrm{~s}<.05]$. However, a comparison with the neutral conditions indicated that the effect of selective attention was greater for the wings-out than for the wingsin figures. The percentage magnitudes for the $60^{\circ}$ and $90^{\circ}$ wings-in selective-attention conditions were $88 \%$ and $86 \%$, respectively, relative to neutral conditions, and 

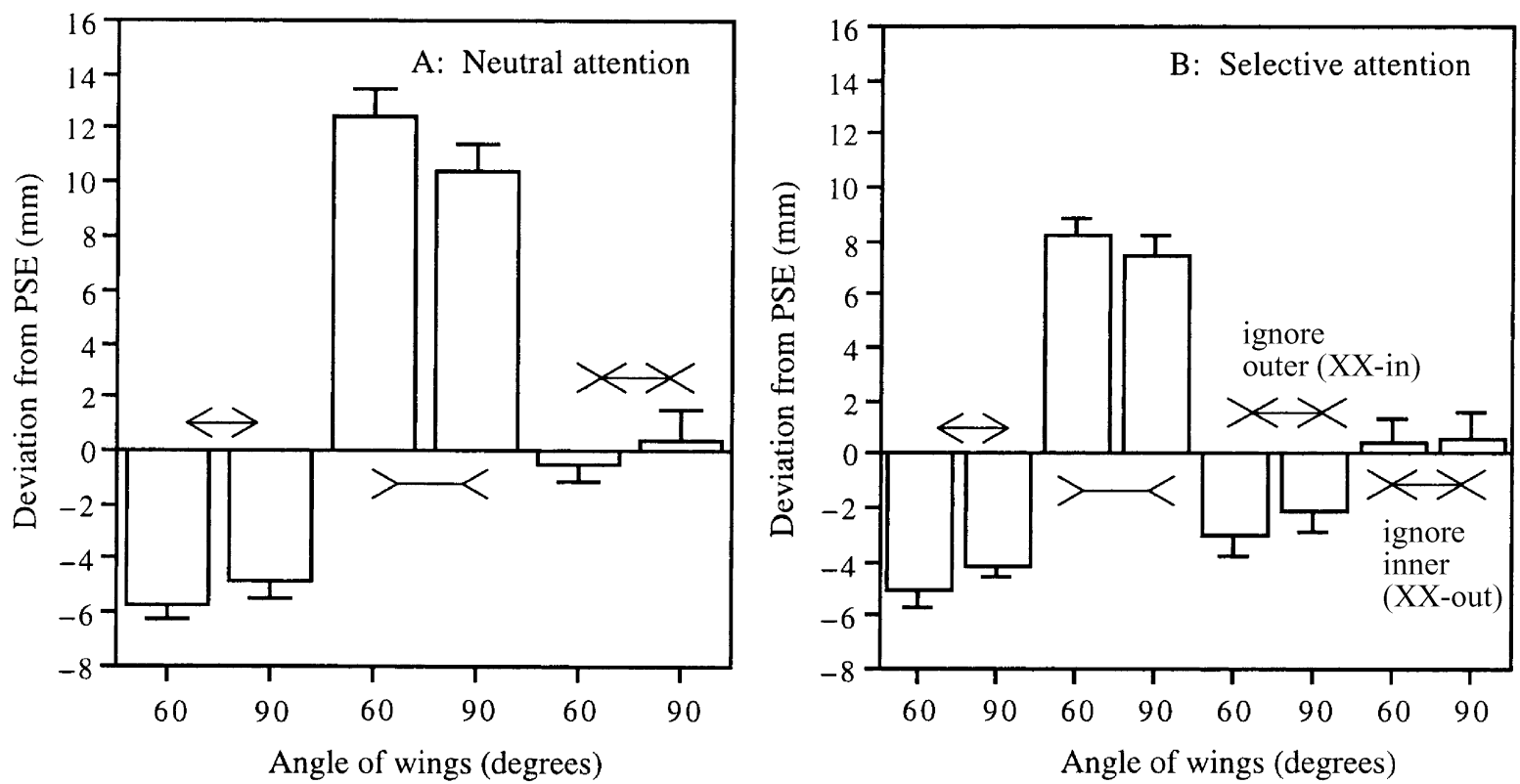

Figure 2. Experiment 1: The magnitudes, in millimeters, of the wings-in, wings-out, and superimposed forms of the MüllerLyer illusion, expressed as deviations from the point of subjective equality (PSE) as a function of internal wing angle. (A) Neutral attention. Subjects judged the apparent length of the shaft for the wings-in $(\ll)$, wings-out $(><$ ), and superimposed $(\lessdot \times)$ forms without ignoring or selectively attending to parts of the figure. (B) Selective attention. Subjects judged the apparent length of the shaft while ignoring the wings of the basic versions $(\leftrightarrow$ and $><$ ) and ignoring either the inner or the outer wings of the superimposed version $(\Varangle x)$.

the corresponding values for the wings-out figures were $65 \%$ and $71 \%$. This reduction was significant for the wings-out figures $[t(15)=4.27$ and 3.02 , respectively; $p s<.05]$, but not for the wings-in figures $[t(15)=1.28$ and 1.53 , respectively; $p \mathrm{~s}>.05]$.

\section{Discussion}

The results of the XX-out condition indicate that subjects were unable to ignore the inner to attend to the outer wings, thereby rendering this condition equivalent to the XX-neutral condition. This failure of selective attention cannot be attributed to a difficulty in segregating the wings-in from the wings-out oblique line elements, since if this were the case the XX-in condition should also have yielded a null outcome instead of the obtained significant underestimation of shaft length.

The underestimations of the $60^{\circ}$ and $90^{\circ} \mathrm{XX}$-in attentional conditions were significantly smaller than the corresponding illusions with wings-in figures in the neutralattention condition $[t(15)=5.41$ and 4.42 , respectively; $p \mathrm{~s}>.05]$ and selective-attention condition $[t(15)=2.79$ and 3.31, respectively; $p \mathrm{~s}>.05$ ]. This difference was consistent with the residual, albeit nonsignificant, overestimation for the wings-out selective-attention condition, suggesting that subjects were unable to completely ignore the outer wings.

The results of several studies (e.g., Sadza \& de Weert, 1984) suggest that the magnitude of the M-L illusion decreases when the obliques and the shaft are different in color. Arguably a component, or even all, of the reduc- tion effected by the instructions to attend to the shaft and to ignore the wings in the M-L figure conditions of the present experiment is attributable to differences in colorblack lines for the neutral and different colored lines for the test stimuli-and not to the attentional manipulation. The purpose of Experiment 2 therefore was to determine whether under the conditions of the present experiments, color differences per se attenuated the two forms of the M-L illusion.

\section{EXPERIMENT 2}

The test stimuli were the black (i.e., baseline) and the two sets of colored M-L stimuli used in Experiment 1. Half the subjects were presented with one of the color sets and half with the other. The test and baseline stimuli were presented in separate blocks, with the block order counterbalanced across subjects. Each block consisted of two randomized presentations of four M-L figures (two angles $X$ two wing types). Prior to the test blocks, subjects were given a block of practice trials consisting of 40-, 50-, and 60 -mm-long plain horizontal lines. The instructions for both the test and the baseline sessions were identical to the neutral-attention instructions used in the baseline condition of Experiment 1. In all other respects, the stimulus dimensions, viewing distance, and procedure were identical to those of Experiment 1. The 13 subjects all had normal or corrected-to-normal vision.

Each subject's length reproductions of the 50-mmlong practice line were used to convert their reproduc- 
tions of the M-L figures to deviations from PSE. Since preliminary analyses indicated no significant differences between the two colored stimulus sets, the data were combined. For the black stimulus figures, the means (with standard errors in parentheses) of the $60^{\circ}$ and $90^{\circ}$ wings-in M-L conditions were $-5.19(0.63)$ and -4.60 $(0.61) \mathrm{mm}$, respectively, and 12.78 (1.14) and 10.87 (0.84) $\mathrm{mm}$ for the corresponding wings-out M-L conditions. For the colored stimulus figures, the means and standard errors of the $60^{\circ}$ and $90^{\circ}$ wings-in M-L conditions were $-5.04(0.48)$ and $-4.12(0.57) \mathrm{mm}$, respectively, and $12.16(0.78)$ and $9.97(0.67) \mathrm{mm}$ for the corresponding wings-out conditions.

None of the four tests comparing the illusion magnitudes of the four colored M-L figure conditions with those of the otherwise identical black M-L figure conditions was significant (all $t \mathrm{~s}<1.35, p\rangle .05$ ). These results suggest that the reduction in the magnitudes of the M-L illusion effected by the selective-attention manipulation in Experiment 1 is unlikely to be caused by color differences between the shaft and wing components. In any event, the reported effects of color differences on M-L illusion magnitudes are quite small. Sadza and de Weert (1984), using the combined M-L figure (Figure 1C), found a difference of about $3.2 \%$ between the illusion magnitudes of one-colored and two-colored M-L stimuli. Because this estimate is based on their subjects comparing the two M-L forms, as distinct from judging each form separately, their results suggest a reduction in magnitude of only about $1.6 \%$ each for the two-colored wings-in and wings-out forms of the M-L illusion.

\section{GENERAL DISCUSSION}

The present findings confirm the importance of the distribution of attention in modulating the strength of the wings-in, wings-out, and superimposed forms of the M-L illusion by demonstrating the modulation of illusion magnitude by varying the spatial focus of visual attention in the absence of any physical change in the stimulus. Additionally, the findings suggest that it is unnecessary to attribute the asymmetrical results between the XX-out and $\mathrm{XX}$-in conditions to a difficulty in perceptually segregating the inner from the outer wings, although the role of such a difficulty when using black-and-white XX figures, as in Tsal's (1984) study, cannot be discounted.

The effectiveness of attentional variations in determining the direction and magnitude of the distortions in XX figures has been interpreted as implicating high-level ("central" or "cognitive") determinants of the M-L illusion. However, space-based approaches to visual attention provide an alternative - perhaps a more parsimonious explanation - of the present results, particularly in view of the difficulty in this and other studies for observers to ignore the inner wings of the $\mathrm{XX}$ figure. According to such approaches, the asymmetry in the illusion magnitudes for the XX-out and XX-in attentional conditions is a result of visual input or "external" factors - that is, to differences in the sampling of stimulus information con- sequent on different patterns of gazing or foveating on the figures elicited by the task requirement to ignore one or the other wing component. From this perspective, attentional effects do not mandate the conclusion that central factors are major determinants of the M-L illusion.

Arguably, the two modes of visual attention-objectversus space-based theories of attention - are not mutually exclusive. Indeed, the possibility that under certain circumstances, observers could ignore the inner wings of XX figures, as might be predicted by object-centered theories, cannot be excluded. For example, extended practice may enhance the ability to segregate the inner wings from the outer wings and shaft, especially if, as in the present experiment, the shaft and outer wings share a feature (e.g., color) that the inner wings do not.

Recent psychophysical and physiological work conducted in a different context from the present investigation indicates that visual attention modulates visual processing, although the locus and nature of attention's effect on processing remains uncertain. Nevertheless, converging evidence is required to substantiate the claim that attention-induced changes in the direction and magnitudes of length distortion for the XX version of the M-L illusion reflect modulations of internal processes responsible for the formation of the M-L illusion rather than external variations in visual input arising from the differential sampling of spatial information.

\section{REFERENCES}

Christie, P. S. (1975). Asymmetry in the Mueller-Lyer illusion: Artifact or genuine effect? Perception, 4, 453-457.

Coren, S., \& Porac, C. (1983). The creation and reversal of the MüllerLyer illusion through attentional manipulation. Perception, 12, 4954.

Eriksen, C. W., \& St. James, J. D. (1986). Visual attention within and around the field of focal attention: A zoom lens model. Perception \& Psychophysics, 40, 225-240.

ErLebacher, A., \& SeKuler, R. (1969). Explanation of the MüllerLyer illusion: Confusion theory examined. Journal of Experimental Psychology, 80, 462-467.

GARDNER, R. W., \& LONG, R. I. (1961). Selective attention and the Müller-Lyer illusion. Psychological Record, 11, 317-320.

Goryo, K., Robinson, J. O., \& Wilson, J. A. (1984). Selective looking and the Müller-Lyer illusion: The effect of changes in the focus of attention on the Müller-Lyer illusion. Perception, 13, 647-654.

Pressey, A. W. (1971). An extension of assimilation theory to illusions of size, area, and direction. Perception \& Psychophysics, 9, 172-176.

Pressey, A. [W.], \& Martin, N. S. (1990). The effects of varying fins in Müller-Lyer and Holding illusions. Psychological Research, $\underline{\mathbf{5 2}}$, 46-53.

Restle, F., \& Decker, J. (1977). Size of the Mueller-Lyer illusion as a function of its dimensions: Theory and data. Perception \& Psychophysics, 21, 489-503.

RocK, I. (1975). An introduction to perception. New York: Macmillan. SAdZA, K. J., \& DE WeERT, C. M. M. (1984). Influence of color and luminance on the Müller-Lyer illusion. Perception \& Psychophysics, 35, 214-220.

SCHOLL, B. J. (2001). Objects and attention: The state of the art. Cognition, 80, 1-46.

TsAL, Y. (1984). A Mueller-Lyer illusion induced by selective attention. Quarterly Journal of Experimental Psychology, 36A, 319-333.

(Manuscript received June 3, 2003;

revision accepted for publication November 3, 2003.) 